\title{
OBJECTIVE QUALITY ASSESSMENT OF IMAGE ENHANCEMENT METHODS IN DIGITAL MAMMOGRAPHY-A COMPARATIVE STUDY
}

\author{
Sheba K.U. ${ }^{1}$ and Gladston Raj S. ${ }^{2}$ \\ ${ }^{1}$ Department of Computer Applications, BPC College, Piravom \\ ${ }^{2}$ Department of Computer Science, Government College, Nedumangad
}

\begin{abstract}
Mammography is the primary and most reliable technique for detection of breast cancer. Mammograms are examined for the presence of malignant masses and indirect signs of malignancy such as micro calcifications, architectural distortion and bilateral asymmetry. However, Mammograms are X-ray images taken with low radiation dosage which results in low contrast, noisy images. Also, malignancies in dense breast are difficult to detect due to opaque uniform background in mammograms. Hence, techniques for improving visual screening of mammograms are essential. Image enhancement techniques are used to improve the visual quality of the images. This paper presents the comparative study of different preprocessing techniques used for enhancement of mammograms in mini-MIAS data base. Performance of the image enhancement techniques is evaluated using objective image quality assessment techniques. They include simple statistical error metrics like PSNR and human visual system (HVS) feature based metrics such as SSIM, NCC, UIQI, and Discrete Entropy
\end{abstract}

\section{KEYWORDS}

CLAHE, HE, Contrast stretching, Wavelet transforms, Adaptive median filter

\section{INTRODUCTION}

Breast cancer is the most common cancer among women worldwide constituting more than $25 \%$ of all cancer incidences occurring in the world [1]. Statistics show that US, India and China account for more than one third of all breast cancer cases [2]. Also, there has been a steady increase in the breast cancer incidence among young generation in the world. In India, one out of two women die after being detected with breast cancer where as in China it is one in four and in USA it is one in eight [2]. Therefore, the statistics show that cancer mortality is highest in India among all other nations in the world. In US, though the number of women diagnosed with cancer is more than that in India, their mortality rate is less due to the early detection of breast cancer and early treatment.

Cancers are the result of uncontrollable growth of cells in the body. Genes which reside in the nucleus of the cells are responsible for the orderly process of cell growth i.e. when old cells die, new cells take their place. But due to mutation or abnormal changes in the genes, the genes lose control of the cell growth. Hence, cells start dividing at an uncontrollable rate resulting in tumour [3].

At present, there are no preventive measures available for breast cancer. Hence early detection and treatment is necessary for reducing breast cancer mortality. According to the US Preventive DOI : 10.5121/sipij.2016.7401 
Signal \& Image Processing : An International Journal (SIPIJ) Vol.7, No.4, August 2016

Services Task (USPST) [4], several screening modalities are available for early detection of breast cancer. They include Mammography, Magnetic Resonance Imaging (MRI), Positron Emission Tomography (PET), breast cancer examination and clinical breast cancer examination.

Among all the screening modalities, mammography is considered to be the most effective method for breast cancer detection [5]. Mammography is a lost cost, low dose X-ray procedure which provides an internal view of the breast parenchyma. A radiologist assessing the mammograms will look for the following type of changes - masses, micro calcifications, bilateral asymmetry and architectural distortion [6].

Mammography though highly reliable is not perfect. This is due to the following reasons.

- Reading mammograms is a demanding job for the radiologists as they may have to evaluate a batch consisting of 100 or more mammograms in one sitting. Only $0.5 \%$ [7] of these mammograms may be abnormal. Chances are that some of the subtle abnormalities may be missed due to the monotonous job.

- The judgment of the radiologists are based on their experience and training. Some of the abnormalities may be missed due to their inexperience in the field.

- In mammograms there are only small differences in the X-ray attenuation of malignant and normal tissues.

- Mammograms of women with dense breast are difficult to analyze, hence chances of missing the detection of malignancies in women with dense tissues is higher.

- Micro calcifications are smaller in size and tend to have low contrast, hence can be missed.

- Mammograms may be of poor quality. Hence, they are difficult to interpret. This may be due to low contrast of the mammograms, presence of artifacts, labels, unknown noise, and weak boundaries and also the presence of unrelated parts like pectoral muscles.

The above problems can be rectified if the radiologists are assisted by breast cancer CAD systems. Computer Aided Diagnosis (CAD) systems [8] integrate computer science with biomedical image analysis and aids the radiologists in making diagnostic decisions with the help of the output from computerized analysis of medical images. Breast Cancer CAD systems help the radiologists by providing second opinion in interpreting mammograms for breast cancer detection and classification. It has been found that detection accuracy without CAD is $80 \%$ and with CAD is 90\% [9]. This is because humans are prone to errors and their evaluation is based on subjective and qualitative analysis where as CAD provides objective and quantitative analysis. $\mathrm{CAD}$ systems involve the following phases-Image acquisition, image pre-processing, image segmentation, feature extraction and selection and classification.

\section{IMAGE PRE-PROCESSING OF MAMMOGRAMS}

The aim of preprocessing is to improve the image quality so as to enhance the perception of information in the images either for human viewing or for some other specific application. Image pre processing involves image enhancement and noise removal.

The aim of preprocessing in mammograms is to enhance the breast profile from the background and to remove the artifacts, labels and other noise from it to produce reliable representation of the breast tissue. So, it is important for the mammograms to undergo processing in order to make it suitable for the remaining phases - segmentation, feature extraction, selection and classification. 
Signal \& Image Processing : An International Journal (SIPIJ) Vol.7, No.4, August 2016

The first step in preprocessing is image enhancement which mainly includes contrast enhancement, intensity manipulation and sharpening, filtering, magnification and so on.

Several techniques have been reported in literature for enhancement which include unsharp masking [10], spatial filtering [11], region based contrast enhancement [12], wavelet transformation [13], histogram equalization [14], morphological operators [15].

The aim of this research is to implement different enhancement techniques on the mammograms and to evaluate each one of them using image quality assessment techniques so as to choose the appropriate enhancement technique. Section 3 specifies materials and methods, Section 4 evaluates the results and Section 5 concludes the paper.

\section{MATERIALS AND METHODS}

The digital mammographic images have been obtained from the mini-MIAS database [16]. The database includes 322 breast images of 161 patients which have been carefully selected, expertly diagnosed and the positions of abnormalities have been recorded in case of malignant and benign mammograms. The mammograms in this database were obtained using medio-lateral oblique view (MLO). They are digitized at a spatial resolution of $0.05 \mathrm{~mm}$ pixel size with 8 bit density resolution using SCANDIG -3. There are 208 normal, 63 benign and 51 malignant images.

\subsection{Image Enhancement Techniques}

All enhancement methods can be grouped into three types - global histogram modification approach, local processing approach and multi scale processing approach [9]. In this paper, five enhancement techniques from all the three types have been applied to the digital mammographic images and have been analyzed.

\subsubsection{Global histogram modification approach}

\subsubsection{Histogram Equalization}

Histogram Equalization is a simple and effective method to obtain an enhanced image using the uniform histogram [19].HE stretches and flattens the dynamic range of the image resulting in the overall contrast enhancement of the image [18].

In most medical images, the region of interest and its background have close contrast values. With better distribution of intensities on the histogram, HE provides better contrast of the image. This method is most useful for medical image applications because it helps to increase the contrast. It helps in better visualization of region of interest from the background.

\subsubsection{Contrast Stretching}

In contrast stretching [19], the contrast of the image is enhanced by adjusting the histogram of the image so that there is a greater separation between the foreground and background gray-level distribution.

In medical images with low contrast, contrast stretching improves the contrast of the image providing better visualization of region of interest. Also, the computational speed is high. 
Signal \& Image Processing : An International Journal (SIPIJ) Vol.7, No.4, August 2016

\subsubsection{Local processing approach}

\subsubsection{Contrast limited adaptive Histogram Equalization}

CLAHE [17] algorithm is used for contrast enhancement. It divides the original image into several non overlapping sub images and the histograms for each are derived. Histograms of sub images are cut at some threshold to limit the amount of enhancement and then equalized. The details of the image appear clearly relative to the background. At the same time, background is also enhanced as the foreground thus leading to a high contrast image [20].

In medical images, CLAHE effectively enhances the local features and the image contrast while suppressing the noise. It also preserves the richness of details and the sharpness of edges. Compared to Histogram Equalization, it takes less computational time.

\subsubsection{Adaptive Median Filter}

The Adaptive Median Filter [29] is designed to eliminate the problems faced with the standard median filter. Adaptive median filters adapt to the properties of the image locally and selectively removes noise from the image while preserving the edges. Thus, the Adaptive Median Filter seeks to preserve detail while smoothing non impulse noise. Adaptive median filtering can handle the filtering operation of an image corrupted with impulse noise of probability greater than 0.2.

Adaptive median filtering is suitable for enhancing medical images as they smooth non repulsive noise from two dimensional signals without blurring edges. They also preserve the details of the image.

\subsubsection{Multiscale processing approach}

\subsubsection{Wavelet based Enhancement}

Wavelet Transform [20] is a powerful tool for filtering images where images are represented hierarchically on the basis of scale and resolution. It decomposes an image onto a set of frequency channels having a constant bandwidth in a logarithmic scale. Variation of resolution enables the wavelet transform to focus on irregularities of a signal and characterize them locally.

The advantage of using wavelet transform for medical image enhancement is that it helps to focus on features of importance. It helps in the visualization of features without amplifying noise. It also improves the visualization of unseen and barely seen features.

\subsection{Image Quality Assessment Techniques}

The goal of image quality assessment technique [22] is to evaluate the perceptual quality of the image. The most accurate evaluation of the image quality is through the eyes of the human observer who makes use of the image. This is known as subjective evaluation. Though it is the most accurate method, it is time consuming, expensive and also depends on the mood, lighting condition, vision ability etc. of the subject. This makes it impractical in real time processing [22].

Objective IQA methods make use of mathematical models that can automatically measure the image quality which is almost at par with the subjective evaluation. There are two classes of objective IQA techniques - simple statistical error metrics and human visual system (HVS) feature based metrics [23]. The former does not take into account whether the enhanced image contains more visual information or not where as the latter takes into account the visual 
Signal \& Image Processing : An International Journal (SIPIJ) Vol.7, No.4, August 2016

information of images. Also, simple statistical error metrics become unstable when the amount of degradation is high. So, HVS feature based metrics provide better subjective assessment.

A few from both classes have been used to assess the quality of enhanced mammograms. In all the metrics defined here, $I_{i j}$ refers to the intensity value of the original image and $E_{i j}$ refers to the intensity value of the enhanced image at $\mathrm{i}$ and $\mathrm{j}$ respectively. $\mathrm{M}$ and $\mathrm{N}$ refers to the width and height of the image.

\subsubsection{Simple Statistical Error Metrics}

\subsubsection{Peak Signal to noise Ratio}

PSNR [24] is a deviation of the current image from the original image with respect to the peak value of the gray level and is given by Equation 1 .

$$
\mathrm{PSNR}=10 \log \frac{M A X^{2}}{M S E}
$$

Here MAX is the possible maximum value in the image usually 255. MSE is the mean squared error difference between the original and enhanced image and is given by equation 2 .

$$
\mathrm{MSE}=\frac{1}{M N} \sum_{i=1}^{M} \sum_{j=1}^{N}\left(E_{i j}-I_{i j}\right)^{2}
$$

Higher the value of PSNR better is the quality of enhanced image.

\subsubsection{Human visual system (HVS) feature based metrics.}

\subsubsection{Structural Similarity Index Metric (SSIM)}

SSIM [25] is a HVS metric for measuring the similarity between the images based on luminance, contrast and structure of images.

$$
\operatorname{SSIM}(\mathrm{I}, \mathrm{E})=\frac{\left(2 \mu_{I} \mu_{E}+C_{1}\right)\left(2 \sigma_{I E}+C_{2}\right)}{\left(\mu_{I}^{2}+\mu_{E}^{2}+C_{1}\right)\left(\sigma_{I}^{2}+\sigma_{E}^{2}+C_{2}\right)}
$$

Here $\mu_{I}=$ Average of I, $\mu_{E}=$ Average of $\mathrm{E}, \sigma_{I}^{2}=$ variance of I, $\sigma_{E}^{2}=$ variance of $\mathrm{E}, \sigma_{I E}=$ Covariance of IE, $C_{1}=\mathrm{K}_{1} \mathrm{~L}^{2}$ is a constant used to avoid instability when $\mu_{I}^{2}+\mu_{E}^{2}$ is close to zero. $\mathrm{K}_{1}=0.01, \mathrm{~L}=255 . C_{2}=\mathrm{K}_{2} \mathrm{~L}^{2}$ is the constant used to avoid instability when $\sigma_{I}^{2}+\sigma_{E}^{2}$ is close to $0 . \mathrm{K}_{2}=0.03, \mathrm{~L}=255$.

The value of SSIM is between $(-1,1)$. When SSIM value is closer to 1 , better is the quality of enhanced image.

\subsubsection{Universal Image Quality Index (UIQI)}

UIQI [26] models image distortion as a combination of three factors- loss of correlation, luminance distortion and contrast distortion. 
Signal \& Image Processing : An International Journal (SIPIJ) Vol.7, No.4, August 2016

$$
\mathrm{UIQI}=\frac{\left(4 * \sigma_{I E}\right)\left(\mu_{I}+\mu_{E}\right)}{\left(\mu_{I}^{2}+\mu_{E}^{2}\right)\left(\sigma_{I}^{2}+\sigma_{E}^{2}\right)}
$$

$\sigma_{I E}=$ Covariance of I and $\mathrm{E}, \mu_{\mathrm{I}}=$ Average of $\mathrm{I}, \mu_{\mathrm{E}}=$ average of $\mathrm{E}, \sigma_{I}^{2}=$ variance of $\mathrm{I}, \sigma_{\bar{E}}^{2}=$ variance of $E$. The value of UIQI ranges from -1 to 1 .

UIQI value of 1 indicates full similarity where as UIQI value of -1 indicates total dissimilarity.

\subsubsection{Normalized Correlation Coefficient (NCC)}

NCC [27] is also an objective evaluation metric used to indicate the similarity between images.

$$
\mathrm{NCC}=\frac{\sum_{i=1}^{M} \sum_{j=1}^{N} I_{i j} * E_{i j}}{\left(\sqrt{\sum_{i=1}^{M} \sum_{j=1}^{N} I_{i j}^{2}}\right)\left(\sqrt{\sum_{i=1}^{M} \sum_{j=1}^{N} E_{i j}^{2}}\right)}
$$

The NCC values ranges between 0 and 1 . NCC value closer to 1 indicates high similarity between images and 0 indicates no similarity.

\subsubsection{Discrete Entropy (DE)}

Discrete Entropy [28] measures the richness of details in an image after enhancement.

$$
\mathrm{E}(\mathrm{I})=-\sum_{k=0}^{255} P\left(I_{k}\right) \log _{2}\left(P\left(I_{k}\right)\right)
$$

Here, $\mathrm{P}\left(\mathrm{I}_{k}\right)$ is the probability density function of the $k^{\text {th }}$ gray level.

Higher value of DE indicates an image with richer details.

\section{RESULTS AND DISCUSSIONS}

322 mammograms belonging to normal, benign and malignant category with fatty, fatty glandular and dense breast tissues were taken as sample mammograms. Each of these mammograms was enhanced using the enhancement techniques discussed in section III. Figure 3 shows a sample mammogram and the enhancement results obtained using HE, CLAHE, Adaptive median filter, DWT and contrast stretching. For the objective evaluation, metrics discussed in section III were used. The comparative analysis of each of the metrics is tabulated in tables 1, 2, 3, 4 and 5. Each table shows the values of metrics obtained for 5 mammograms chosen from all categories. The breast image mdb001 is a fatty glandular benign type, mdb021 is fatty-glandular malignant breast image, mdb058 is dense malignant image, mdb063 is dense benign breast image and mdb070 is fatty normal breast image. The last row of each table shows the average value of the metric obtained for all the 322 images. The corresponding bar charts with average values of metrics obtained for 322 mammograms are also provided.

The values of PSNR measure is tabulated in table 1. It is clear from table 1 and figure 3, the value of PSNR for adaptive median filter is higher than all other enhancement techniques which indicate better quality. 
The values of SSIM are tabulated in table 2.It is clear from table 2 and figure 2 SSIM value is closer to 1 for adaptive median filter followed by contrast stretching indicating that adaptive median filter provides maximum similarity.

The values of NCC in table 3 show that it is closest to 1 for adaptive median filter.

The UIQI values in table 5 show that it is above 0.95 for adaptive median filter and thus is superior compared to other methods.

The entropy value is highest for DWT followed closely by CLAHE and adaptive median filter in Table 4. Higher values show that the richness of details in original image is preserved by DWT, CLAHE and adaptive median filter after enhancement.

Enhancement techniques like contrast stretching and HE enhanced the contrast more than it was desired. This proved as a disadvantage in case of dense breasts. Dense breasts in mammograms have brighter areas. These brighter areas make the detection of malignancies difficult especially micro calcifications since they are small in size and have low contrast. Adaptive median filter and DWT in general allowed the reduction of bright areas in dense mammograms thus making it possible to identify malignancies.

HE took high computational time as compared to other techniques, so it proved to be a major disadvantage.

Adaptive Median filter is the superior enhancement technique as far as providing better quality, maximum similarity and preserving richness of details is concerned.

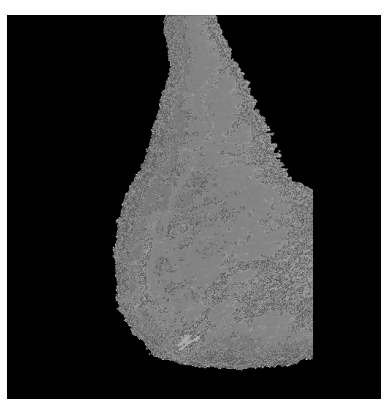

(a)

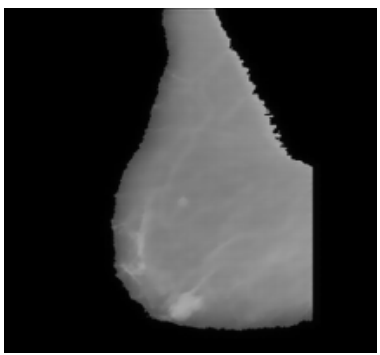

(d)

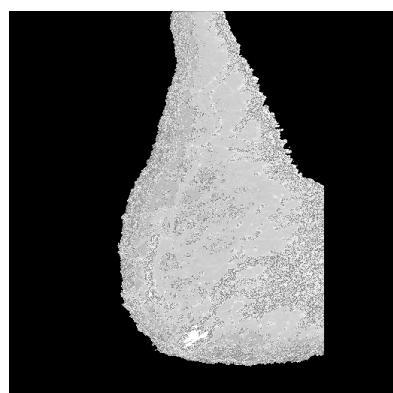

(b)

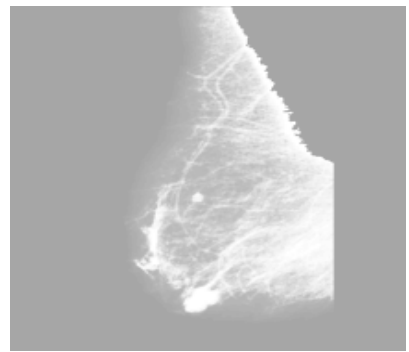

(e)

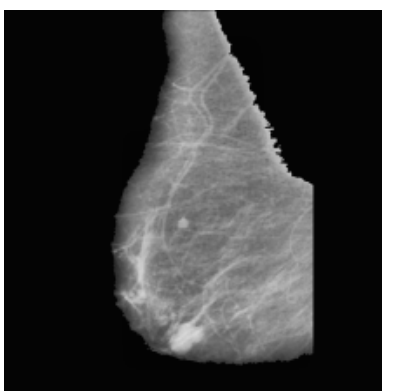

( c)

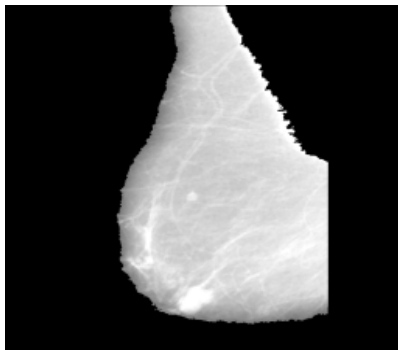

( f)

Figure1. Sample image and enhanced images.(a) sample image (b) enhanced image with adaptive median filter (c) enhanced image with CLAHE (d) enhanced image with wavelet transform (e) enhanced image with HE (f) enhanced image with contrast stretching 
Signal \& Image Processing : An International Journal (SIPIJ) Vol.7, No.4, August 2016

Table 1 PSNR values for 5 mammograms

\begin{tabular}{|c|l|l|c|c|c|}
\hline & HE & CLAHE & $\begin{array}{l}\text { Adaptive } \\
\text { median }\end{array}$ & $\begin{array}{l}\text { Contrast } \\
\text { Stretching }\end{array}$ & DWT \\
\hline mdb001 & 5.316192 & 28.30375 & 34.37068 & 15.29862 & 23.47135 \\
\hline mdb021 & 6.404888 & 27.44357 & 36.83815 & 15.05533 & 23.83456 \\
\hline mdb058 & 4.046295 & 26.5409 & 37.35696 & 17.65433 & 23.61625 \\
\hline mdb063 & 4.655262 & 25.4469 & 36.4197 & 16.91583 & 24.82495 \\
\hline mdb070 & 3.592979 & 29.4935 & 30.71567 & 28.73049 & 20.56182 \\
\hline $\begin{array}{c}\text { Average value for 322 } \\
\text { images }\end{array}$ & 4.6002 & 27.23226 & 35.76972 & 19.27183 & 23.4506 \\
\hline
\end{tabular}

Table 2 SSIM values for 5 mammograms

\begin{tabular}{|c|l|l|l|l|l|}
\hline & HE & CLAHE & $\begin{array}{l}\text { Adaptive } \\
\text { median }\end{array}$ & $\begin{array}{l}\text { Contrast } \\
\text { Stretching }\end{array}$ & \multicolumn{1}{l|}{ DWT } \\
\hline mdb001 & 0.288715 & 0.54348 & 0.990869 & 0.958947 & 0.815682 \\
\hline mdb021 & 0.330038 & 0.57541 & 0.993064 & 0.960409 & 0.808798 \\
\hline mdb058 & 0.240502 & 0.483998 & 0.993134 & 0.97638 & 0.833721 \\
\hline mdb063 & 0.275574 & 0.502763 & 0.992237 & 0.971759 & 0.812741 \\
\hline mdb070 & 0.217382 & 0.497904 & 0.985287 & 0.997155 & 0.807046 \\
\hline $\begin{array}{c}\text { Average value for } 322 \\
\text { images }\end{array}$ & 0.261449 & 0.517717 & 0.990683 & 0.967406 & 0.81447 \\
\hline
\end{tabular}

Table 3 NCC values for 5 mammograms

\begin{tabular}{|c|c|c|c|c|c|}
\hline & HE & CLAHE & $\begin{array}{c}\text { Adaptive } \\
\text { median }\end{array}$ & $\begin{array}{c}\text { Contrast } \\
\text { Streching }\end{array}$ & DWT \\
\hline mdb001 & 0.665745 & 0.795981 & 0.998758 & 0.790603 & 0.988625 \\
\hline mdb021 & 0.697562 & 0.31296 & 0.999386 & 0.597696 & 0.992898 \\
\hline mdb058 & 0.578212 & 0.983774 & 0.999228 & 0.891104 & 0.987768 \\
\hline mdb063 & 0.962042 & 0.955232 & 0.999109 & 0.605038 & 0.996172 \\
\hline mdb070 & 0.443486 & 0.775501 & 0.996664 & 0.19376 & 0.957494 \\
\hline $\begin{array}{c}\text { Average value for 322 } \\
\text { images }\end{array}$ & 0.706676 & 0.652817 & 0.998718 & 0.586771 & 0.980237 \\
\hline
\end{tabular}


Signal \& Image Processing : An International Journal (SIPIJ) Vol.7, No.4, August 2016

Table 4 ENTROPY values for 5 mammograms

\begin{tabular}{|c|c|c|l|r|l|}
\hline & HE & CLAHE & $\begin{array}{l}\text { Adaptive } \\
\text { median }\end{array}$ & $\begin{array}{l}\text { Contrast } \\
\text { Stretching }\end{array}$ & DWT \\
\hline mdb001 & 2.548606 & 3.590208 & 3.116364 & 3.048539 & 3.661045 \\
\hline mdb021 & 2.823665 & 3.959229 & 3.417423 & 3.358782 & 3.920546 \\
\hline mdb058 & 2.02719 & 3.059476 & 2.683792 & 2.639243 & 3.137768 \\
\hline mdb063 & 2.243136 & 3.375814 & 2.963723 & 2.91391 & 3.460664 \\
\hline mdb070 & 1.807994 & 2.915169 & 2.452837 & 2.406715 & 3.241961 \\
\hline $\begin{array}{c}\text { Average value for } \\
\text { 322 images }\end{array}$ & 2.263691 & 3.361622 & 2.930195 & 2.880412 & 3.497212 \\
\hline
\end{tabular}

Table 5 UIQI values for 5 mammograms

\begin{tabular}{|c|c|c|c|c|c|}
\hline & HE & CLAHE & $\begin{array}{c}\text { Adaptive } \\
\text { median }\end{array}$ & $\begin{array}{c}\text { Contrast } \\
\text { Stretching }\end{array}$ & DWT \\
\hline mdb001 & 0.19409 & 0.237757 & 0.964685 & 0.928677 & 0.508506 \\
\hline mdb021 & 0.198409 & 0.265779 & 0.955599 & 0.928741 & 0.468886 \\
\hline mdb058 & 0.194928 & 0.187702 & 0.98239 & 0.959431 & 0.608306 \\
\hline mdb063 & 0.223533 & 0.215681 & 0.979822 & 0.952581 & 0.562676 \\
\hline mdb070 & 0.162457 & 0.198351 & 0.970329 & 0.989873 & 0.545411 \\
\hline $\begin{array}{c}\text { Average value for } \\
322 \text { images }\end{array}$ & 0.196735 & 0.217024 & 0.969934 & 0.943715 & 0.539035 \\
\hline
\end{tabular}

SSIM VALUES

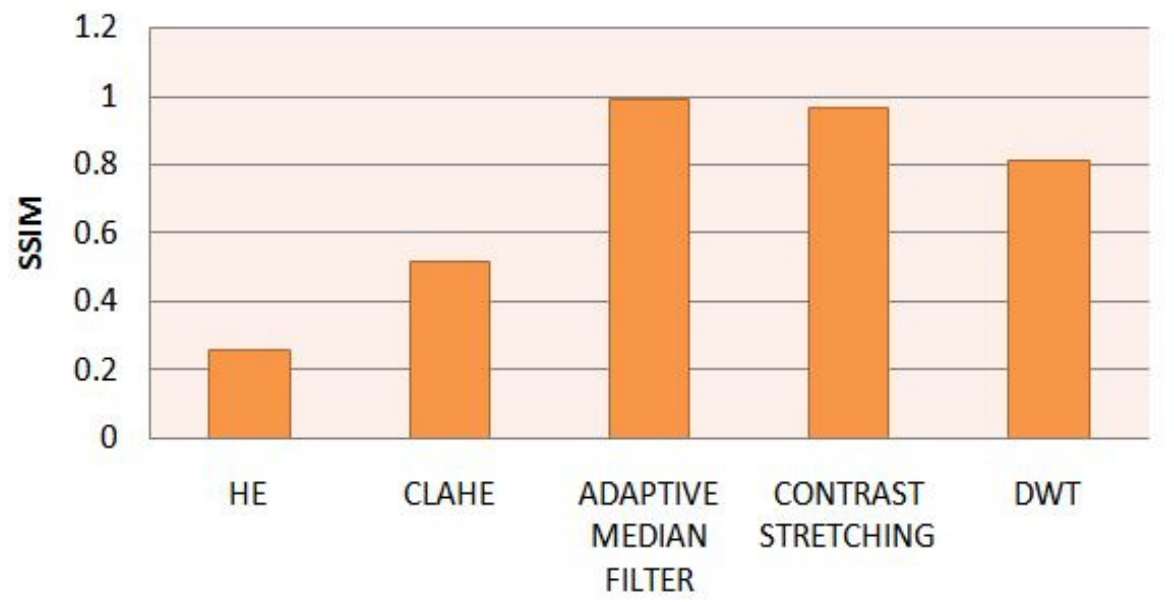

Figure 2 SSIM Values 
Signal \& Image Processing : An International Journal (SIPIJ) Vol.7, No.4, August 2016

\section{PSNR VALUES}

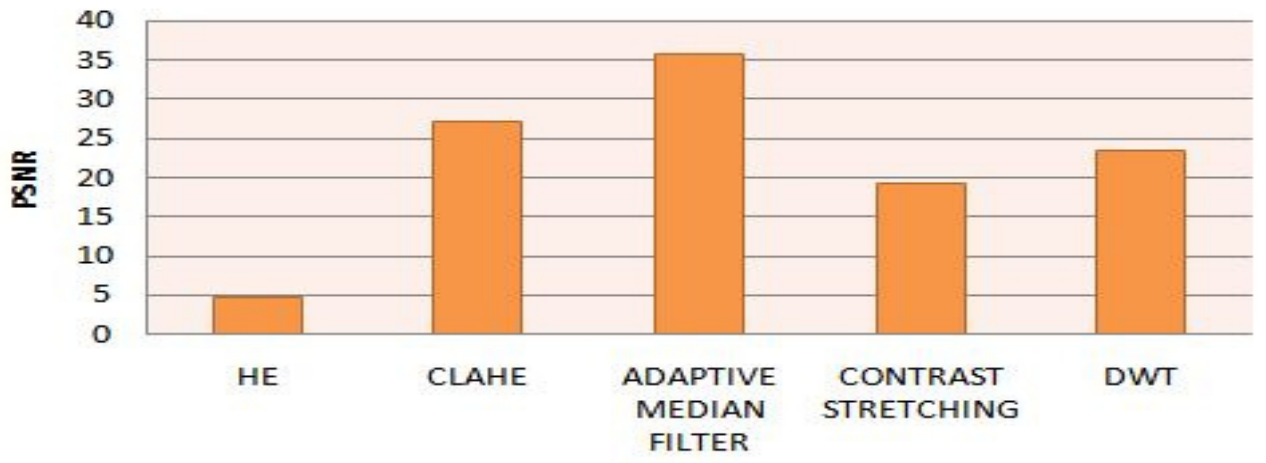

Figure 3 PSNR Values.

\section{ENTROPY VALUES}

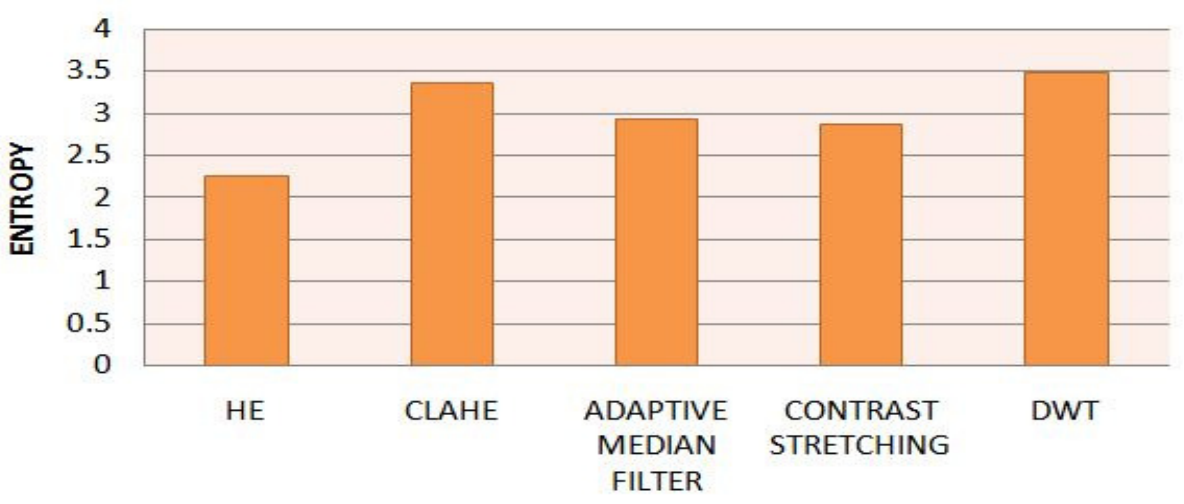

Figure 4 Entropy values

NCC VALUES

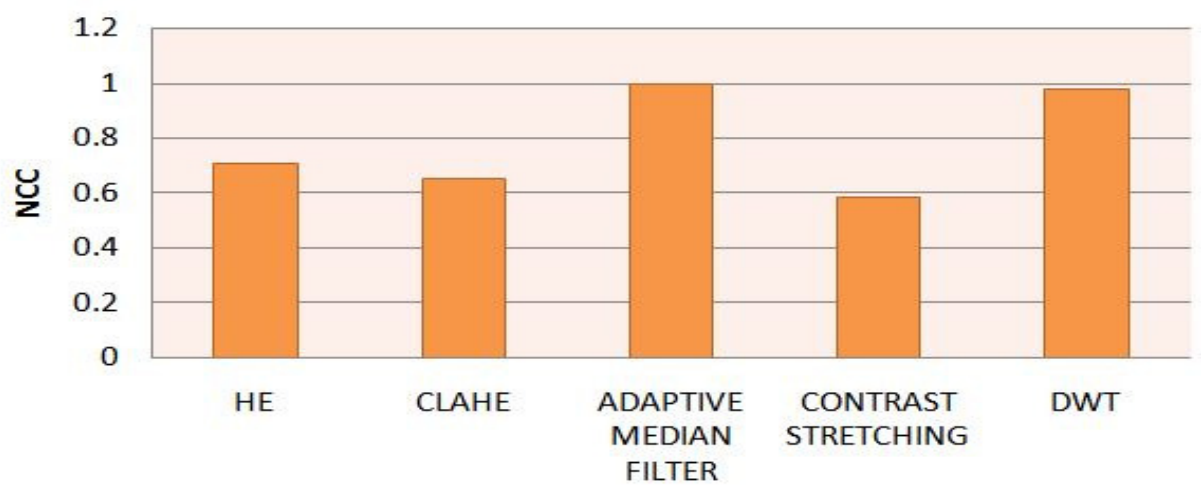

Figure 5 NCC values 


\section{UIQI VALUES}

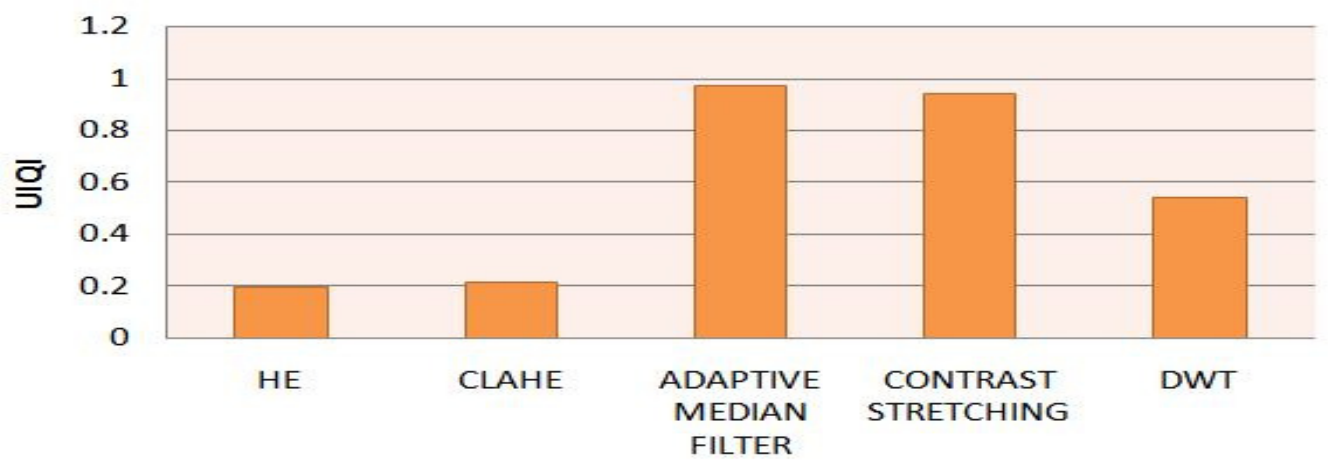

Figure 6 UIQI values

To demonstrate the efficacy of adaptive median filter, spatial filtering is applied on the enhanced images. Average filter, wiener filter and LOG are applied. The results are analyzed using PSNR, NCC and SSIM. The quantitative analysis of these metrics is tabulated in tables 6,7 and 8 respectively. The results of spatial filtering indicate the robustness of adaptive median filter.

Table 6 Performance Comparison on Filtered Image with NCC measure

\begin{tabular}{|c|c|c|c|c|c|}
\hline & HE & CLAHE & $\begin{array}{c}\text { Adaptive } \\
\text { Median }\end{array}$ & $\begin{array}{c}\text { Contrast } \\
\text { Stretching }\end{array}$ & DWT \\
\hline LOG & 0.24726 & 0.99651 & 0.99744 & 0.996813 & 0.99455 \\
\hline Average & 0.24736 & 0.99865 & 0.99906 & 0.998761 & 0.99787 \\
\hline Weiner & 0.24737 & 0.99869 & 0.99972 & 0.999672 & 0.99771 \\
\hline
\end{tabular}

Table 7 Performance Comparison on Filtered Image with PSNR measure

\begin{tabular}{|c|c|c|c|c|c|}
\hline & HE & CLAHE & $\begin{array}{c}\text { Adaptive } \\
\text { Median }\end{array}$ & $\begin{array}{c}\text { Contrast } \\
\text { Stretching }\end{array}$ & DWT \\
\hline LOG & 4.44623 & 37.9225 & 39.511 & 34.40997 & 34.0394 \\
\hline Average & 4.44555 & 45.1374 & 47.4717 & 41.70384 & 41.6621 \\
\hline Weiner & 4.44431 & 44.6594 & 53.6244 & 48.19623 & 38.4994 \\
\hline
\end{tabular}

Table 8 Performance Comparison on Filtered Image with SSIM measure

\begin{tabular}{|c|c|c|c|c|c|}
\hline & HE & CLAHE & $\begin{array}{c}\text { Adaptive } \\
\text { Median }\end{array}$ & $\begin{array}{c}\text { Contrast } \\
\text { Stretching }\end{array}$ & DWT \\
\hline LOG & 0.24841 & 0.98146 & 0.99323 & 0.987322 & 0.86481 \\
\hline Average & 0.25167 & 0.9965 & 0.99884 & 0.997606 & 0.97859 \\
\hline Weiner & 0.24495 & 0.98115 & 0.99661 & 0.989807 & 0.90458 \\
\hline
\end{tabular}

\section{CONCLUSION}

In this paper, five different enhancement techniques have been implemented and assessed using metrics belonging to both classes of objective IQA techniques.322 mammograms belonging to normal, benign and malignant category with fatty, fatty glandular and dense breast tissues were taken as sample mammograms. The results of the study illustrated that adaptive median filter is 
Signal \& Image Processing : An International Journal (SIPIJ) Vol.7, No.4, August 2016

the most appropriate method compared to others because it provided better quality images, maximum similarity between original and enhanced images and also preserved the richness of details. Adaptive median filter and DWT, in general, allowed the reduction of bright areas in dense mammograms thus making it possible to identify subtle malignancies. Further, the robustness of the adaptive median filter is tested using spatial filtering on the enhanced images. The results of the spatial filtering show the efficacy of adaptive median filter.

\section{REFERENCES}

[1] World cancer research fund international, Cancer facts and figures. Available from http://www.wcrf.org

[2] Statistics of breast cancer in India, Global comparison, available from http://www.breastcancerindia.net

[3] http://www.breastcancer.org/symptoms/understand-bc/what-is-bc

[4] U.S preventive services Task Force, Final Recommendation Statement: Breast Cancer screening available from http://www.uspreventiveservicetaskforce.org.

[5] http://www.cancer.gov/,The American college of Radiology(ACR), http://www.acr.org.

[6] Sheba K.U, Gladston Raj S," Latest Advances in Diagnosis of Breast cancer using image processing techniques in Digital Mammograms", International Journal Of Advanced Research in Biology, Engineering, Science and Technology (IJARBEST), volume 1, special issue 5 - December 2015, pp.32-36.

[7] R.J Brenner, AJR 156: 719-723, 1991.

[8] R.M Rangayyan, Bio medical Image Analysis, CRC press LLC, Boca Raton, Florida, USA, 2005.

[9] H.D. Cheng, X.J Shi, R. Min, LM Hu, XP Cai, H.N. Du, "Approaches for automated detection and classification of masses in mammograms", pattern recognition, 39 (2006), 646-668

[10] Panetta K, Zhou Y, Agaian S, Jia H.," Non linear unsharp masking for mammogram enhancement",. IEEE Transactions on information technology in Bio medicine 2011, 15 (6): 918-28.

[11] P.G Tahoces, J. Correa, M. Souto, C Gonzalez, L. Gomez and J.J Vidal "Enhancement of chest and breast radiographs by automatic spatial filtering", IEEE Trans. Med. Imag. Vol 10, No. 3, pp. 330335.

[12] Morrow WM, Paranjape RB, Rangayyan RM, "Region based contrast enhancement of mammograms", IEEE Trans Med imaging. 1992. 11(3): 392-406.

[13] Mencattini A, Salmeri M, Lojacono, Frigerio R, Caselli MF, "Mammographic Images enhancement and denoising for breast cancer detection using Dyadic wavelet processing", IEEE Transactions on instrumentation and measurement 2008, 57(7): 1422-30.

[14] Gandhi K.R, Karnan M.,” Mammogram image enhancement and segmentation”, IEEE international conference on computational intelligence and computing research (ICCIC) 2010-1:1-4

[15] Hassanien A E, Abraham A., "Rough Morphology hybrid approach for Mammography image classification and prediction", International journal of computational intelligence and applications 2008 7(1): 17-42.

[16] http://peipa.esser.ac.uk/pix/mias/ 
Signal \& Image Processing : An International Journal (SIPIJ) Vol.7, No.4, August 2016

[17] Pisano, Etta D, Elodia Cole, Bradley M. Hemminger, Maria Deluca, Keith Muller, M. Patrick Braeuning, Stephen M. Pizer, "Contrast limited adaptive histogram equalization image processing to improve detection of simulated spiculations in dense mammograms", Journal of digital imaging II, No. 4, 1998: 193-200.

[18] Pisano, Etta D, Elodia Cole, Bradley M. Hemminger, Martin J. Yaffe et.al,'Image processing algorithms for digital Mammography- A pictorial Essay", I. Radiographics 20, No. 5. 2000: 14791491.

[19] A.K. Jain, Fundamentals of digital Image processing, Eaglewood cliffs, NJ prentice Hall, 1989.

[20] I. Daubechies, "Orthogonal bases of compactly supported wavelets", Communication pure and applied Mathematics, Vol 41, no 11, pp 909-916, 1988.

[21] Tang J, Liu X, Sun Q, “A Direct Image contrast enhancement Algorithm in the wavelet Domain for screening Mammograms”, IEEE Journal of selected topics in signal processing 2009. 3(1):74.

[22] Pedram Mohammadi, Abbas Ebrahim - Mogha dam, Shahram Shirani ,"Subjective and objective quality assessment of image: A survey", Majlesi Journal of Electrical Engineering, Vol (9) No. 1 (2015)

[23] Z. Wang, A.C Bovik., Modern Image Quality Assessment. Morgan and Claypool publishing company, New York, 2006.

[24] Rajkumar Soundrapadiyan, Chandra Mouli, Springer-verlag Berlin Heidelberg, Trans. on Computer Science XXV, LNCS 9030, pp 3-19, 2015.

[25] Wang Z, Bovik A.C, Sheikh HR, Simoncelli E.P, "Image Quality Assessment from error visibility to structural similarity”, IEEE Trans. Image processing 13(4), 600-612 (2004)

[26] Wang Z, Bovik A.C,” A Universal Image Quality Index”,. Signal processing letter 9(3), 81-84 (2002)

[27] Lewis J.P,” Fast Normalized cross-correlation vis interface”, 10(1), 120-123 (1995)

[28] P. Shanmugavadivu, K. Balasubramanian, K. Somasundaram, "Modified Histogram Equalization for Image Contrast Enhancement using particle swarm optimization", International Journal of Computer Science, Engineering and Information Technology, Vol 1, No. 5, December 2011.

[29] R.C. Gonzalez, R.E. Woods, Digital Image Processing, Prentice-Hall Inc., 2006. 